\title{
Supporting Wiki-Based Knowledge Exchange Processes
}

\author{
Sven Heimbuch (advised by Daniel Bodemer) ${ }^{1}$ \\ ${ }^{1}$ University of Duisburg-Essen, Germany \\ Corresponding author: Sven Heimbuch \\ Email address: sven.heimbuch@uni-due.de
}

\section{ABSTRACT}

Complex knowledge exchange processes in collaborative knowledge building settings within wikis can be either supported by providing guidance in form of cognitive group awareness information or by explicitly guiding learners with collaboration scripts. My Ph.D. project comprises a series of three experimental studies to determine which kind of support is most beneficial for varying types of learners working with wikis. For this research different fields of CSCL are integrated and both quantitative and qualitative methods are applied to provide comprehensive analyses in order to provide opportunities for other related research. Presenting and discussing aspects of my research and first results could be beneficial for my future research.

\section{BACKGROUND}

Due to the structures of common wikis (e.g. Wikipedia using MediaWiki), collaborative knowledge building within such environments especially focused on knowledge exchange via article talk pages as a basis for discussions can be a challenging task for participants. This Ph.D. project's overarching goals are (1) to quantitatively and qualitatively analyse knowledge exchange processes in wikis used in educational contexts, (2) to analyse and support communication processes between authors and editors beneficially for learning, and (3) to develop and evaluate wiki modifications for more effective and efficient collaboration and learning by structuring relevant aspects of collaborative knowledge building.

Knowledge building can be defined as the creation of knowledge as a social product (Scardamalia and Bereiter, 1994). A significant amount of research has been conducted on how knowledge building and in consequence learning processes can be backed by computer-supported collaborative learning (CSCL) environments like online discussion forums, blogs or wikis. Collaborative knowledge building can lead to controversies and furthermore to socio-cognitive conflicts. Such conflicts arising from contradictory information do not have to be detrimental for learning (Mugny and Doise, 1978).

Supportive measures for dealing with conflicts that have proven to be effective for learners in CSCL in different contexts range from implementations of implicit guidance approaches, e.g. implementation of cognitive group awareness tools (Janssen and Bodemer, 2013), to more explicit instructional methods, e.g. instructional designs through collaboration scripts (Dillenbourg, 2002). Wikis differ fundamentally from "classic"online discussion forums that have been analysed more extensively because of its two layer distinction of article view and the corresponding discussions. However, little research has been conducted specifically on those wiki article talks as a layer for knowledge exchange.

Therefore, it is of particular interest how participants in informal wiki learning settings can be further supported with implicit and/or explicit guiding aids to benefit from socio-cognitive conflicts arising from controversies that are led by opposing evidences. In addition to that, my doctoral research covers the influences of specific cognitive and personality constructs that can be relevant for learning processes. These constructs are an individual's (1) 
need for cognitive closure and (2) epistemic curiosity that both are potential mediators for successful learning and should be considered for further implementations and design recommendations.

For this Ph.D. project a number of three experimental studies have been planned to analyse support mechanisms for different types of learners in CSCL settings. In the first study, an implicit structuring aid as cognitive group awareness tool was implemented to support learners to focus on relevant evidenceled controversies rather than onto non-contentual discussions (Heimbuch and Bodemer, 2014). In a second study, that has to be analysed in detail yet, I was particularly interested in the comparison of two different collaboration script approaches (Heimbuch et al., 2014).

\subsection{Experimental Scenario}

All experimental studies implement varying degrees and types of learners support (implicit vs. explicit vs. combined guidance), focussed on informal learning via conflicting information provided on wiki talk pages. Learning materials are differing in topics (e.g. mass extinction of dinosaurs, pirate personalities etc.). As an important common ground all studies comprise content-related controversies that are led by evidence, i.e. relevant research, rather than personal opinions.

For the first study, a single independent factor with three levels was randomly varied across the study. The three experimental groups reflect differing implicit structuring degrees of additionally implemented cognitive group awareness support on controversy information for a number of 24 wiki talk page discussions. Study participants in the two supported conditions were primarily focussed on meaningful and relevant discussions in order to complete the task of editing a basic wiki article by themselves. A total of $N=81$ university students (58 females and 23 males), aged 18 to 30 ( $M=$ $27.70, S D=2.76$ ), were randomly assigned to the three experimental groups.

\section{FIRST STUDY}

In either of the guidance groups (G2 / G3), students preferred to select and read the most relevant topics containing evidence-led conflicting discussions at first. In contrast, participants without additional guidance (G1) showed the tendency to follow a less focussed top-down reading strategy. This first look at most frequent closed sequential patterns indicates that guidance towards the potentially most relevant discussions of interest worked as intended in both guidance groups. These results are further supported by reviewing the students' topic clicking behaviours. A corresponding analysis of variance revealed that in $\mathrm{G} 1$ significantly more topics on the article's talk page were selected, compared to both supported conditions G2 / G3 $(F(2,78)=3.80, p=$ $\left..027, \eta^{2}=.09\right)$, indicating a more focussed selection and reading behaviour by providing implicit guidance. Further analysis of variance on the learning success, measured by a multiple-choice knowledge test, could not reveal any overall differences between the three investigated groups, $F(2,78)=0.03$, $p=.968, \eta^{2}<.01$. More detailed analyses of the knowledge test scores between all groups revealed, considering different categories of discussion types as mediators in a parallel multiple single-step mediation, students receiving more implicit guidance (G3 > G2) and spending more time on reading unsolved controversies scored significantly higher in the multiple choice test.

Overall, these results indicate that structuring wiki talk pages by implementing cognitive group awareness representations related to socio-cognitive conflicts produced promising results in terms of focussing readers' attention towards relevant. Furthermore, the results demonstrate that implicitly guiding readers towards relevant evidence-led discussions containing opposing points of view leads to measurably higher learning success.

\section{REFERENCES}

Dillenbourg, P. (2002). Over-scripting CSCL: The risks of blending collaborative learning with instructional design. Three worlds of CSCL. Can we support CSCL?, pages 61-91. 
Heimbuch, S. and Bodemer, D. (2014). Supporting awareness of content-related controversies in a wiki-based learning environment. In Proceedings of The International Symposium on Open Collaboration, pages 30:1-4, New York, NY, USA. ACM.

Heimbuch, S., Uhde, K., and Bodemer, D. (2014). "A new Wiki way?" - An experimental study of collaborative knowledge building scripts. In Liu, C.-C. et al., editor, Proceedings of the 22nd International Conference on Computers in Education, volume WIPP, pages 10-12, Nara, Japan. AsiaPacific Society for Computers in Education.

Janssen, J. and Bodemer, D. (2013). Coordinated computer-supported collaborative learning: Awareness and awareness tools. Educational Psychologist, 48(1):40-55.

Mugny, G. and Doise, W. (1978). Socio-cognitive conflict and structure of individual and collective performances. European Journal of Social Psychology, 8(2):181-192.

Scardamalia, M. and Bereiter, C. (1994). Computer support for knowledge-building communities. Journal of the Learning Sciences, 3(3):265283. 